\title{
A new filter for the Mean Dynamic Topography of the ocean derived directly from satellite observations
}

\author{
G. Freiwald ${ }^{a}$ \\ ${ }^{a}$ Alfred Wegener Institute for Polar and Marine Research, Postfach 120161, 27515 \\ Bremerhaven, Germany
}

\begin{abstract}
The Mean Dynamic Topography (MDT) of the ocean provides valuable information about the ocean's surface currents. Therefore the MDT is computed from satellite observations and then assimilated into ocean models in order to improve the ocean circulation estimates. However, the computation of the MDT from satellite observations of sea surface height and the Earth's gravity field is not straightforward and requires additional filtering of the data combination. The choice of the filter is crucial as it determines the amount of small-scale noise in the data and the resolution of the final MDT. There exist various approaches for the determination of an "optimal" filter. However, they all have in common the more or less subjective choice of the filter type and filter width. Here, a new filter is presented that is determined directly from the geodetic normal equations. By its construction, this filter accurately accounts for the correlations within the MDT data and requires no subjective choice about the filter radius. The new filtered MDT is assimilated into an inverse ocean model. Modifications in the meridional overturning circulation and in the poleward heat transports can be observed, compared to the result
\end{abstract}


of the assimilation using the unfiltered MDT.

Keywords: Filter, Mean Dynamic Topography of the ocean, Error covariance estimate, Inverse ocean models

\section{Introduction}

The Mean Dynamic Topography (MDT) of the ocean is the difference

3 between the Mean Sea Surface height and the geoid height, the geoid being

4 an equipotential surface of the Earth's gravity field. The computation of the

5 MDT is not straightforward because the different observational data sets have

6 different representations and different resolution (Becker et al., 2012; Losch

7 et al., 2002). Therefore, filtering becomes necessary in the MDT computation

8 to remove small-scale noise.

9 Different approaches exist for the choice of the required filter ${ }^{1}$ (Jekeli, 10 1981; Bingham et al., 2008; Jayne, 2006). A common choice is a Gaussian 11 filter with an appropriate half-width radius. In Knudsen et al. (2011), a 12 method is described for the determination of an "ideal" Gaussian filter width. 13 Bosch and Savcenko (2009) promote an along-track filtering approach for the altimetric data and tolerate filter errors that arise from this one-dimensional filtering. An anisotropic filter is also used in Bingham et al. (2011) to filter the MDT. Filters that account for the error correlations of gravity field data are constructed e.g. in Swenson and Wahr (2006) and Kusche (2007). However, at the current stage, it is not clear which filtering is the most appropriate for the MDT.

\footnotetext{
${ }^{1}$ 'Filter' is used here in terms of mapping an input signal onto an output signal. It is not used in terms of LTI systems.
} 
In this study, we use the MDT error covariance matrix for the construction of a filter for the MDT data. The development of an MDT filter based on error covariances was already suggested in Bingham et al. (2008), however, its implementation depends on the availability of such an error covariance matrix. Here, the MDT estimate and its corresponding dense error covariance matrix described in Becker et al. (2012) are used.

The paper is organized as follows. An introduction to the MDT estimate and an introduction to the ocean model IFEOM are given in sections 2.1 and 2.2, respectively. The derivation of the new filter is illustrated in section 3.1. The filtered MDT and the filter residuals are compared to the results obtained by another filtering type in section 3.2. The assimilation of the new filtered MDT into the ocean model IFEOM and a comparison of the results to those of the assimilation of the unfiltered MDT are presented in section 4. A concluding discussion is provided in section 5 .

\section{Background}

\subsection{Mean Dynamic Topography}

The Mean Dynamic Topography (MDT) can be used to estimate ocean surface currents via the principle of geostrophy. Hence the combination of satellite observations of the sea surface height and the gravity field can reveal valuable information about the ocean's circulation (Wunsch and Stammer, 1998). However, satellite data of the MDT can only provide an incomplete picture of the ocean's state due to its two-dimensionality. Therefore in this study an MDT estimate is combined with an inverse ocean model in order to improve the understanding of the ocean's three-dimensional mean circulation. 
57 cost function

$$
J=\frac{1}{2} \sum_{i} J_{i} \stackrel{!}{=} \min , \quad \text { where } i=\text { MDT, temperature, salinity, etc. }
$$
Becker et al. (2012). This MDT is designed exclusively for inverse ocean model assimilation. The MDT data $\boldsymbol{\eta}_{\mathrm{d}}$ and its corresponding inverse error covariance matrix $\boldsymbol{C}^{-1}$ are computed directly on an ocean model grid. The inverse error covariance is estimated from a least squares adjustment (geodetic normal equations) as described in Becker et al. (2012). This dense inverse MDT error covariance matrix is used as weighting matrix for the MDT model-data misfit in the ocean model optimization.

\subsection{Inverse Finite Element Ocean Model (IFEOM)}

The Inverse Finite Element Ocean Model (IFEOM) is a stationary model for the North Atlantic ocean (Sidorenko et al., 2006). It combines physical principles with observational data such as in-situ temperature and salinity measurements and satellite data. This is accomplished by minimizing the

For this purpose, a MDT was estimated from satellite observations by

The different terms $J_{i}$ contain quadratic model-data differences weighted by the inverses of their respective error covariances. Contributions from the residuals of the advection-diffusion equations for temperature and salinity are also contained in the cost function, so that the residuals are small. In this study, temperature and salinity data from a hydrographic atlas (Gouretski and Koltermann, 2004) are used for all IFEOM model runs. The MDT and its inverse error covariance matrix (section 2.1) are assimilated in an unfiltered and in a filtered version. 
In general, error correlations are unknown and diagonal inverse "covariance" matrices are used for weighting the different cost function terms. In our case, the full dense inverse error covariance matrix $\boldsymbol{C}^{-1}$ for the MDT data $\eta_{\mathrm{d}}$ is provided by the approach described in Becker et al. (2012). Therefore the MDT term in the cost function (1) reads

$$
J_{\mathrm{MDT}}=\left(\boldsymbol{\eta}_{\mathrm{d}}-\boldsymbol{\eta}_{\mathrm{m}}\right)^{T} \alpha^{-1} \boldsymbol{C}^{-1}\left(\boldsymbol{\eta}_{\mathrm{d}}-\boldsymbol{\eta}_{\mathrm{m}}\right) .
$$

\begin{abstract}
with the "observed" MDT $\boldsymbol{\eta}_{\mathrm{d}}$ from satellite data and their modeled counterparts $\boldsymbol{\eta}_{\mathrm{m}}$. The scalar factor $\alpha$ is derived from the Minimum Penalty Variance (MPV) approach (Freiwald, 2012) and is required for additional scaling.

The cost function (1) is minimized iteratively, starting from a first guess which is an earlier IFEOM solution described in Richter (2010). This first guess was computed using only the hydrographic data (temperature and salinity as described above), and therefore it is used here for a comparison with the model runs which assimilate MDT information. Details of IFEOM can be found in Sidorenko (2004) and Freiwald (2012).
\end{abstract}

\title{
3. A new filter based on the inverse error covariance
}

\subsection{Construction}

The inverse MDT error covariance matrix $\boldsymbol{C}^{-1}$ (section 2.1) is used to construct the filter in order to account for the correlations in the MDT data.

In a first step, the matrix square root of $\boldsymbol{C}^{-1}$ is computed. This is possible and unambiguous because the inverse error covariance matrix is positive definite and symmetric by definition. In a second step, each row $i$ of the resulting matrix $\boldsymbol{C}^{-\frac{1}{2}}$ is normalized. The corresponding normalization factors 
88

(not eigenvalues!) $d_{i}$ are used to build the diagonal matrix $\boldsymbol{D}$ :

$$
C^{-\frac{1}{2}}=D \cdot S
$$

89

For the computation of this decomposition, it has to be guaranteed that the diagonal entries $d_{i}$ do not vanish. Due to the structure of the commonly used covariance matrices, this generally applies in applications: The covariance matrices have very large diagonals exceeding the off-diagonals by magnitudes, and therefore also the inverse and the inverse square root of a typical covariance matrix meet the condition.

The resulting matrix $\boldsymbol{S}$ from equation (3) has rows normalized to give a sum of one. This is necessary because the matrix $\boldsymbol{S}$ will be used to filter the MDT data $\boldsymbol{\eta}_{\mathrm{d}}$. The normalization ensures that the MDT is not reinforced or attenuated by the filtering process. This is equivalent to a weighted moving average filter with the weights given by the rows of $\boldsymbol{S}$, thus derived from the error covariances.

The unfiltered MDT $\boldsymbol{\eta}_{\mathrm{d}}$ and the filtered MDT $\boldsymbol{S} \boldsymbol{\eta}_{\mathrm{d}}$ are shown in figure 1. Small-scale noise ("stripes") is largely removed by the filter $\boldsymbol{S}$ while oceanographic structures associated with strong currents, e.g. the Gulf Stream, are not considerably attenuated.

\subsection{Comparison to simple moving average filters}

In order to illustrate the advantage of this covariance-dependent filtering method, a comparison to the results computed with a simple moving average filter is performed. Figure 2 shows the satellite MDT $\boldsymbol{\eta}_{\mathrm{d}}$ filtered with simple moving averages of different radii. It is obvious from the figures that a filter width of $1.0^{\circ}$ or $1.5^{\circ}$ latitude/longitude is not sufficient to eliminate the 

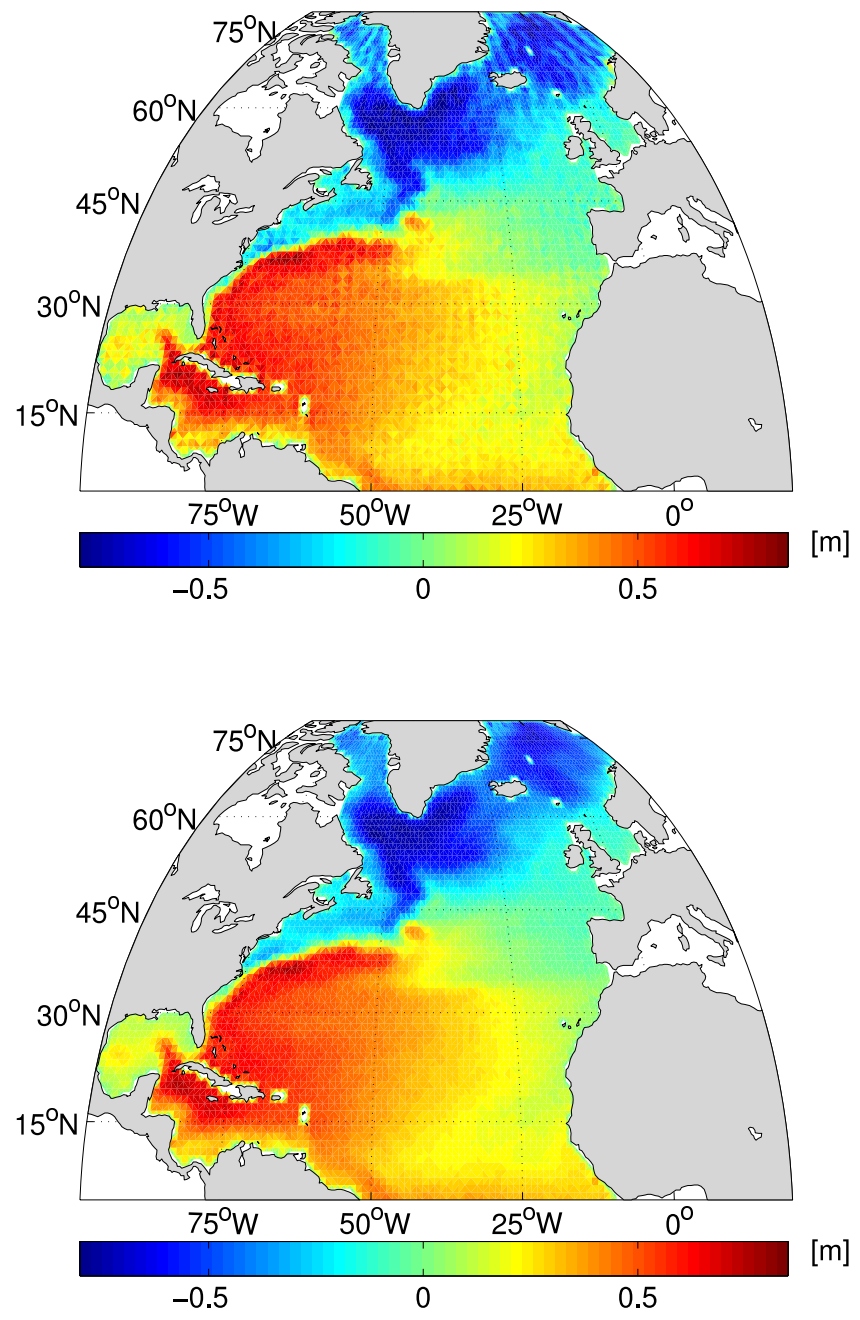

Figure 1: Unfiltered satellite MDT estimate $\boldsymbol{\eta}_{\mathrm{d}}$ (top) and filtered MDT estimate $\boldsymbol{S} \boldsymbol{\eta}_{\mathrm{d}}$ (bottom) 

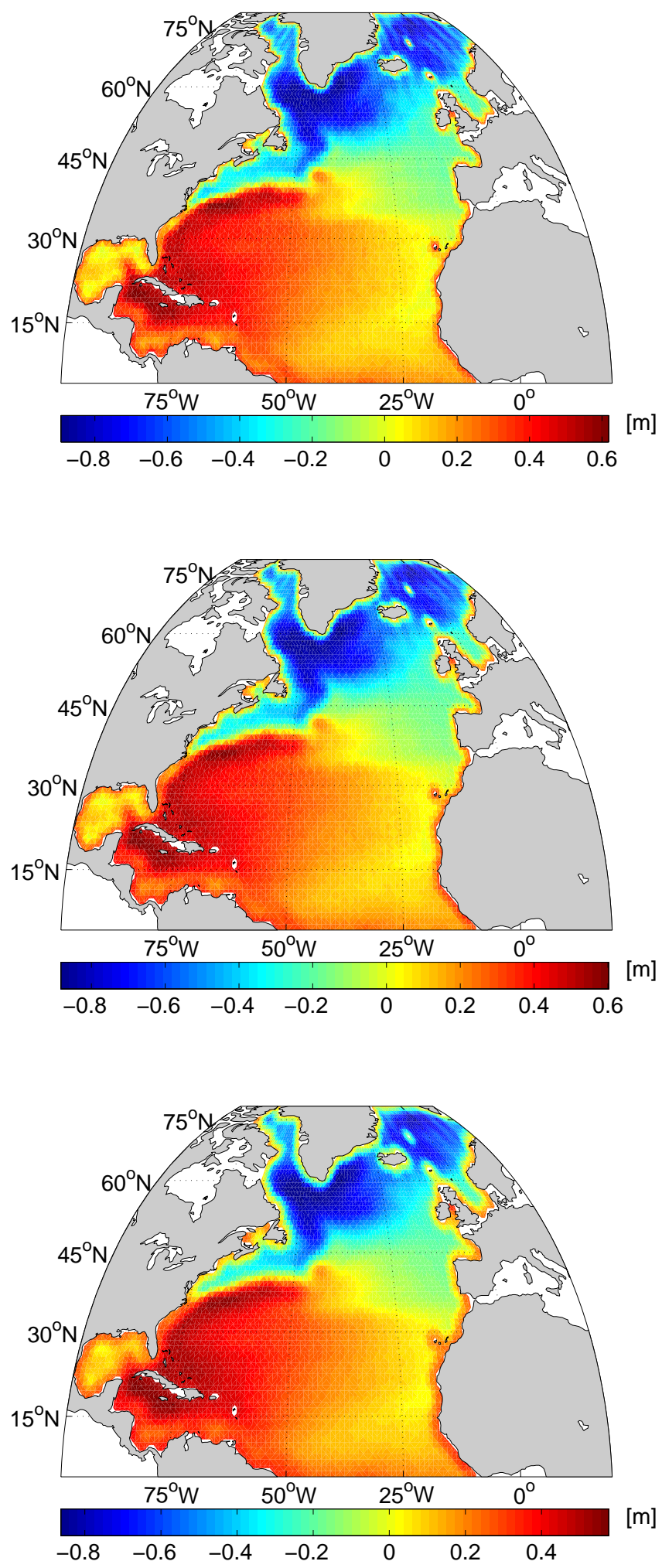

Figure 2: Satellite MDT estimate $\boldsymbol{\eta}_{\mathrm{d}}$ filtered with a simple moving average filter of radius $1^{\circ}$ latitude/longitude (top), $1.5^{\circ}$ (middle) and $2^{\circ}$ (bottom) 
longitudinal "stripes" in the Nordic Seas. Hence, at least a filter width of $2^{\circ}$ latitude/longitude is required to efficiently remove the noise. Such a large filter radius however leads to blurring of other circulation features, e.g. the North Atlantic current, the Mann eddy at approximately $40^{\circ} \mathrm{N}, 40^{\circ} \mathrm{W}$ and the Loop Current in the Gulf of Mexico, see figure 2 (bottom). The filter width is crucial for the result, but chosen more or less subjectively in most cases. In contrast, the proposed filter $\boldsymbol{S}$ does not require any subjective decisions.

The difference between the unfiltered and the filtered MDT, the filter residual $\boldsymbol{\eta}_{\mathrm{d}}-\boldsymbol{S} \boldsymbol{\eta}_{\mathrm{d}}$, is displayed in figure 3 (top). Noise is effectively removed from the MDT data by the filter $\boldsymbol{S}$. Application of a simple moving average filter of radius $2^{\circ}$ latitude/longitude modifies the circulation much more than the proposed filter $\boldsymbol{S}$, and oceanographic regions with large gradients such as the North Atlantic currents are strongly affected by the simple moving average filter (figure 3, bottom).

In case the MDT data is filtered by a simple moving average filter, it is not clear what the appropriate weighting matrix is in the subsequent ocean model assimilation. In contrast, using the filter $\boldsymbol{S}$ leads to a well-defined weighting matrix. Its derivation is described in the following.

\section{Assimilation into IFEOM}

The MDT estimate described in section 2.1 was designed for the use in the inverse ocean model IFEOM. Therefore, it is now investigated how the result of the ocean model changes when the filtered MDT data are assimilated.

For this purpose, the appropriate weighting matrix for the filtered MDT 

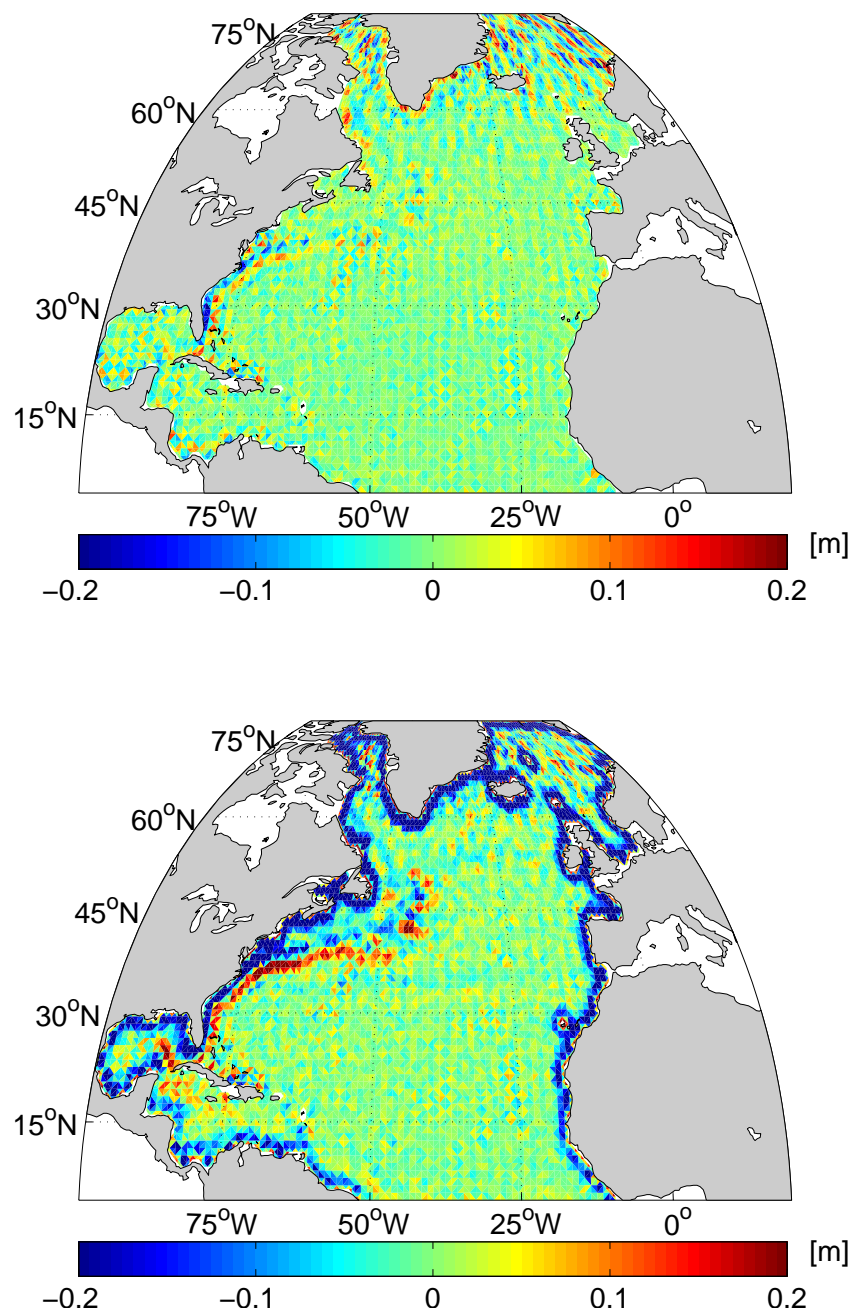

Figure 3: Filter residuals $\boldsymbol{\eta}_{\mathrm{d}}-\boldsymbol{S} \boldsymbol{\eta}_{\mathrm{d}}$ for the filter $\boldsymbol{S}$ (top), and for the simple moving average filter of radius $2^{\circ}$ (bottom) 
data $\boldsymbol{S} \boldsymbol{\eta}_{\mathrm{d}}$ is required. For its determination, we go back to the estimation of $\boldsymbol{\eta}_{\mathrm{d}}$ and $\boldsymbol{C}^{-1}$ from the geodetic normal equations.

The geodetic observation equations are constructed as

$$
\boldsymbol{A} \eta_{\mathrm{d}}=\boldsymbol{l}+\boldsymbol{v}
$$

with a system matrix $\boldsymbol{A}$ that connects the MDT data $\boldsymbol{\eta}_{\mathrm{d}}$ with the observations $\boldsymbol{l}$ (e.g. altimetry and gravity data from satellites) subject to an error $\boldsymbol{v}$ with an observation error covariance $\boldsymbol{V}$. The design of this system of equations is further detailed in Becker (2012). A Generalized Least Squares approach (Draper and Smith, 1998) is made to solve the system:

$$
\underbrace{\boldsymbol{A}^{T} \boldsymbol{V}^{-1} \boldsymbol{A}}_{=: \boldsymbol{C}^{-1}} \boldsymbol{\eta}_{\mathrm{d}}=\underbrace{\boldsymbol{A}^{T} \boldsymbol{V}^{-1} \boldsymbol{l}}_{=: \boldsymbol{n}}
$$

Finally, the normal equation

$$
\boldsymbol{C}^{-1} \boldsymbol{\eta}_{\mathrm{d}}=\boldsymbol{n}
$$

is solved for $\boldsymbol{\eta}_{\mathrm{d}}$ and the matrix $\boldsymbol{C}^{-1}$ is used as weighting matrix in the subsequent ocean model assimilation.

Now the procedure is repeated with the same observations. The only difference is an identity matrix $\boldsymbol{I}=\boldsymbol{S}^{-1} \boldsymbol{S}$ that is introduced into the observation equations:

$$
A \underbrace{S^{-1} S}_{=I} \eta_{\mathrm{d}}=l+v
$$

The Generalized Least Squares approach is applied again, now considering 
$\boldsymbol{S} \boldsymbol{\eta}_{\mathrm{d}}$ as the data and $\boldsymbol{A} \boldsymbol{S}^{-1}$ as the system matrix:

$$
\begin{aligned}
\left(\boldsymbol{A} \boldsymbol{S}^{-1}\right)^{T} \boldsymbol{V}^{-1} \boldsymbol{A} \boldsymbol{S}^{-1} \boldsymbol{S} \boldsymbol{\eta}_{\mathrm{d}} & =\left(\boldsymbol{A} \boldsymbol{S}^{-1}\right)^{T} \boldsymbol{V}^{-1} \boldsymbol{l} \\
\boldsymbol{S}^{-T} \underbrace{\boldsymbol{A}^{T} \boldsymbol{V}^{-1} \boldsymbol{A}}_{=\boldsymbol{C}^{-1}} \boldsymbol{S}^{-1} \boldsymbol{S} \boldsymbol{\eta}_{\mathrm{d}} & =\boldsymbol{S}^{-T} \underbrace{\boldsymbol{A}^{T} \boldsymbol{V}^{-1} \boldsymbol{l}}_{=\boldsymbol{n}} \\
\boldsymbol{S}^{-T} \boldsymbol{C}^{-1} \boldsymbol{S}^{-1} \boldsymbol{S} \boldsymbol{\eta}_{\mathrm{d}} & =\boldsymbol{S}^{-T} \boldsymbol{n} .
\end{aligned}
$$

It follows from the definition of the filter $\boldsymbol{S}$ in equation (3) that the inverse error covariance matrix $\boldsymbol{C}^{-1}$ can be decomposed into

$$
\boldsymbol{C}^{-1}=\boldsymbol{S}^{T} \boldsymbol{D}^{2} \boldsymbol{S}
$$

(This is not equal to an eigenvalue decomposition or to a singular value decomposition.)

Inserting this into equation (10) results in:

$$
\boldsymbol{D}^{2} \boldsymbol{S} \boldsymbol{\eta}_{\mathrm{d}}=\boldsymbol{S}^{-T} \boldsymbol{n}
$$

This system could theoretically be solved for the filtered MDT $\boldsymbol{S} \boldsymbol{\eta}_{\mathrm{d}}$. However, $\boldsymbol{S} \boldsymbol{\eta}_{\mathrm{d}}$ is already known, and equation (12) provides the sought-after weighting matrix $\boldsymbol{D}^{2}$ for the filtered MDT.

Therefore, with the filtered MDT data, IFEOM uses the modified cost function term:

$$
\widehat{J_{\mathrm{MDT}}}=\left(\boldsymbol{S} \boldsymbol{\eta}_{\mathrm{d}}-\boldsymbol{\eta}_{\mathrm{m}}\right)^{T}(\alpha)^{-1} \boldsymbol{D}^{2}\left(\boldsymbol{S} \boldsymbol{\eta}_{\mathrm{d}}-\boldsymbol{\eta}_{\mathrm{m}}\right) .
$$

Note that in equation (13), the filter $\boldsymbol{S}$ is applied only to the MDT observations $\boldsymbol{\eta}_{\mathrm{d}}$. This is in contrast to the ususal approach in equation (2) where $\boldsymbol{C}^{-1}=\boldsymbol{S}^{T} \boldsymbol{D}^{2} \boldsymbol{S}$ and thus both the observational MDT $\boldsymbol{\eta}_{\mathrm{d}}$ and the modeled MDT $\boldsymbol{\eta}_{\mathrm{m}}$ (or the difference of both) are filtered. 
The resulting Atlantic Meridional Overturning Circulation (AMOC) patterns of the two model-data combinations are shown in figure 4 . In the result of the IFEOM model run with the unfiltered MDT, the AMOC is very strong compared to other estimates, which are not shown here, e.g. Griffies et al. (2009); Wunsch (2002); Kuhlbrodt et al. (2007); Hunt (2011). When the filtered MDT is assimilated, the AMOC is decreased and a distinct AMOC maximum is reached at around $40^{\circ} \mathrm{N}$ (figure 4, right). This agrees better with the previous estimates.

From the IFEOM results, also poleward heat transports can be computed. They are presented and compared to other estimates in figure 5. The meridional heat transports agree best with other estimates when the filtered MDT data are used for assimilation. However, these previous estimates are based on different methods (observations/models) and on different time periods, limiting the significance of the comparison. This study does not argue any of the previous studies being superior to another one, but it gives an additional estimate.

Due to boundary effects, the performance of the ocean model IFEOM is very weak at latitudes smaller than approximately $15^{\circ} \mathrm{N}$, and therefore nothing may be evidenced from the result at these low latitudes.

IFEOM provides decent heat transport estimates for the Atlantic basin north of $60^{\circ} \mathrm{N}$. As observations are particularly sparse at these high latitudes, there are hardly any heat transport estimates available to compare our modeled results with. In this situation, the different processing methods for the MDT data set at least provide a possible range of solutions.

It is important to notice that the decreased AMOC and the decreased 

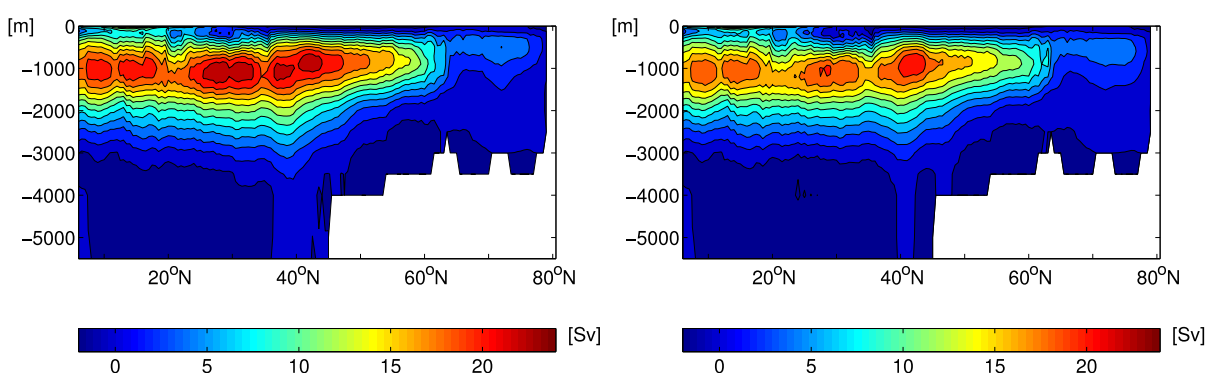

Figure 4: Atlantic Meridional Overturning Circulation (AMOC) by IFEOM using $\boldsymbol{\eta}_{\mathrm{d}}$ and $\boldsymbol{C}^{-1}$ (left) and $\boldsymbol{S} \boldsymbol{\eta}_{\mathrm{d}}$ and $\boldsymbol{D}^{2}$ (right) in the assimilation

heat transports in the model run using the filtered MDT compared to the model run using the unfiltered MDT are not a consequence of possibly weaker gradients in the filtered MDT. The main reason for less adaptation of the model towards the observational data and thus for decreased ocean model circulation strength is the modified weighting matrix $\boldsymbol{D}^{2}$ that goes along with the filtered MDT $\boldsymbol{S} \boldsymbol{\eta}_{d}$. The results of the new method may well be different for other observational data sets. This exemplary study does not provide a general statement or proof.

\section{Summary and discussion}

A new filter for the MDT was developed directly from satellite observations of sea surface height and gravity. The geodetic normal equation matrix was used for the construction of the filter to account for error correlations. No additional assumptions about the type of filter or the filter radius were required. It is a weighted moving average filter with weights computed from the satellite observations. The new filter smoothes the MDT data without considerably attenuating sharp gradients of the MDT. 




Figure 5: Meridional heat transports by IFEOM for a model run without MDT data and for the combined model runs with unfiltered MDT $\boldsymbol{\eta}_{\mathrm{d}}$ and weighting matrix $\boldsymbol{C}^{-1}$ and with the filtered MDT $\boldsymbol{S} \boldsymbol{\eta}_{\mathrm{d}}$ and weighting matrix $\boldsymbol{D}^{2}$. Other estimates include the error ranges from Klein et al. (1995), Lavín et al. (2003), Macdonald and Wunsch (1996), Sato and Rossby (2000), Lorbacher and Koltermann (2000), Bacon (1997) and Lumpkin and Speer (2007) 
A different inverse error covariance is required for the assimilation of the filtered MDT into an inverse ocean model. It follows from the normal equations that the appropriate weighting matrix is the diagonal matrix $\boldsymbol{D}^{2}$ as derived in section 4 . Thus the corresponding error covariance matrix for the filtered MDT is diagonal, meaning all information about the covariances have been shifted into the MDT data themselves. This is equivalent to a transformation of variables as described in Draper and Smith (1998). They transform a correlated set of variables requiring a Generalized Least Squares procedure into a set of variables whose errors are normally distributed. Here, the correlated MDT observations are transformed into uncorrelated ones, however with differing error variances and therefore requiring a Weighted Least Squares approach. So far, it was undiscovered that this transformation can be used as a filter for the MDT data.

The filtered MDT data set $\boldsymbol{S} \boldsymbol{\eta}_{\mathrm{d}}$ was assimilated into the ocean model IFEOM using the weighting matrix $\boldsymbol{D}^{2}$ and the result was compared to the assimilation of the unfiltered data $\boldsymbol{\eta}_{\mathrm{d}}$ with the weighting matrix $\boldsymbol{C}^{-1}$. It was shown that the filtered data set performed better in terms of selected oceanographic features of the resulting model-data combination. The estimates of the $\mathrm{AMOC}$ and the meridional heat transports were decreased compared to those of the assimilation using the unfiltered MDT. Using the filtered MDT in the assimilation increases the agreement with other author's estimates of the AMOC and the meridional heat transports.

From this study, it can be recommended to use this type of filter for satellite MDT data and for subsequent ocean model assimilation. However, the construction of the filter is limited by the availability of a dense inverse 
MDT error covariance estimate. Furthermore, when the MDT data set is large, the matrix square root of a large dense inverse error covariance matrix is required. This may become a computational challenge.

\section{Acknowledgements}

The MDT estimate used for this study was developed within the project "Rifugio" funded by the Deutsche Forschungsgemeinschaft (DFG) priority programme (SPP) 1257 "Mass transport and mass distribution in the system Earth". The MDT data including their error description were provided by Silvia Becker. The author would like to thank Martin Losch, Corinna Ziemer and two anonymous reviewers for their helpful comments that improved the manuscript considerably.

\section{References}

Bacon, S., 1997. Circulation and Fluxes in the North Atlantic between Greenland and Ireland. J. Phys. Oceanogr. 27, 1420-1435.

Becker, S., 2012. Konsistente Kombination von Schwerefeld, Altimetrie und hydrographischen Daten zur Modellierung der dynamischen Ozeantopographie. Ph.D. thesis. Universität Bonn.

Becker, S., Freiwald, G., Losch, M., Schuh, W.D., 2012. Rigorous Fusion of Gravity Field into Stationary Ocean Models. J. Geodyn. 59-60, 99-110. doi:10.1016/j.jog.2011.07.006.

Bingham, R., Haines, K., Hughes, C., 2008. Calculating the Ocean's Mean 
Dynamic Topography from a Mean Sea Surface and a Geoid. J. Atmos. Oceanic Technol. 25. doi:10.1175/2008JTECHO568.1.

Bingham, R., Knudsen, P., Andersen, O., Pail, R., 2011. An initial estimate of the North Atlantic steady-state geostrophic circulation from GOCE. Geophys. Res. Lett. 38. Doi:10.1029/2010GL045633.

Bosch, W., Savcenko, R., 2009. Absolute dynamic ocean topography profiles. Poster, OST Science Team Meeting 22-24 June 2009, Seattle.

Draper, N., Smith, H., 1998. Applied Regression Analysis. John Wiley \& Sons. $3^{\text {rd }}$ edition.

Freiwald, G., 2012. Combining Stationary Ocean Models and Mean Dynamic Topography Data. Ph.D. thesis. Universität Bremen.

Gouretski, V., Koltermann, K., 2004. WOCE Global Hydrographic Climatology. Technical Report. Bundesamt für Seeschifffahrt und Hydrographie, Hamburg und Rostock, Germany.

Griffies, S., Biastoch, A., Böning, C., Bryan, F., Danabasoglu, G., Chassignet, E., England, M., Gerdes, R., Haak, H., Hallberg, R., Hazeleger, W., Jungclaus, J., Large, W., Madec, G., Pirani, A., B.L., S., Scheinert, M., Gupta, A., Severijns, C., Simmons, H., Treguier, A., Winton, M., Yeager, S., Yin, J., 2009. Coordinated Ocean-ice Reference Experiments (COREs). Ocean Model. 26, 1-46.

Hunt, B., 2011. Wind forcing of the ocean and the Atlantic meridional overturning circulation. Clim. Dynam. 37, 19-34. doi:10.1007/s00382-0100860-9. 
Jayne, S., 2006. Circulation of the North Atlantic Ocean from altimetry and the Gravity Recovery and Climate Experiment geoid. J. Geophys. Res. 111. doi:10.1029/2005JC003128.

Jekeli, C., 1981. Alternative methods to smooth the Earth's gravity field. Reports of the Department of Geodetic Science 327. Ohio State University (OSU).

Klein, B., Molinari, R., Müller, T., Siedler, G., 1995. A transatlantic section at 14.5N: Meridional volume and heat fluxes. J. Mar. Res. 53, 929-957.

Knudsen, P., Bingham, R., Anderson, O., Rio, M.H., 2011. A global mean dynamic topography and ocean circulation estimation using a preliminary GOCE gravity field model. J. Geod. Published online, doi: 10.1007/s00190011-0485-8.

Kuhlbrodt, T., Griesel, A., Montoya, M., Levermann, A., Hofmann, M., Rahmstorf, S., 2007. On the driving processes of the Atlantic meridional overturning circulation. Rev. Geophys. 45. doi:10.1029/2004RG000166.

Kusche, J., 2007. Approximate decorrelation and non-isotropic smoothing of time-variable GRACE-type gravity field models. J. Geod. 81, 733-749.

Lavín, A., Bryden, H., Parrilla, G., 2003. Mechanisms of heat, freshwater, oxygen and nutrient transports and budgets at $24^{\circ} \mathrm{N}$ in the Subtropical North Atlantic. Deep-Sea Res. 1, 1099-1128.

Lorbacher, K., Koltermann, K., 2000. Subinertial variability of transport estimates across " $48^{\circ} \mathrm{N}$ " in the North Atlantic. International WOCE Newsletter , 3-5. 
Losch, M., Sloyan, B., Schröter, J., Sneeuw, N., 2002. Box inverse models, altimetry and the geoid: Problems with the omission error. J. Geophys. Res. 107, 15-1-15-13.

Lumpkin, R., Speer, K., 2007. Global Ocean Meridional Overturning. J. Phys. Oceanogr. 37, 2550-2562.

Macdonald, A., Wunsch, C., 1996. An estimate of global ocean circulation and heat fluxes. Nature 382, 436-439.

Richter, F., 2010. Nutzung von Argo-Driftern und Satellitenaltimetriedaten zur Ableitung der Zirkulation im Nordatlantik. Ph.D. thesis. Universität Bremen.

Sato, O., Rossby, T., 2000. Seasonal and Low-Frequency Variability of the Meridional Heat Flux at $36^{\circ} \mathrm{N}$ in the North Atlantic. J. Phys. Oceanogr. 30, 606-621.

Sidorenko, D., 2004. The North Atlantic circulation derived from inverse models. Ph.D. thesis. Universität Bremen.

Sidorenko, D., Danilov, S., Kivman, G., Schröter, J., 2006. On the use of a deep pressure gradient constraint for estimating the steady state ocean circulation from hydrographic data. Geophys. Res. Lett. 33. doi:10.1029/2005GL024716.

Swenson, S., Wahr, J., 2006. Post-processing removal of correlated errors in GRACE data. Geophys. Res. Lett. 33. Doi:10.1029/2005GL025285. 
319 Wunsch, C., 2002. What Is the Thermohaline Circulation? Science 298, $320 \quad 1179-1181$.

321 Wunsch, C., Stammer, D., 1998. Satellite altimetry, the marine geoid, and 322 the oceanic general circulation. Annu. Rev. Earth Pl. Sc. 26, 219-253. 